\title{
Trapped-ion Fock-state preparation by potential deformation
}

\author{
M. A. Simón $\odot,{ }^{1}$ M. Palmero $\odot,{ }^{2}$ S. Martínez-Garaot, ${ }^{1}$ and J. G. Muga $\odot^{1}$ \\ ${ }^{1}$ Department of Physical Chemistry, UPV/EHU, Apdo. 644, Bilbao 48080, Spain \\ ${ }^{2}$ Science and Math Cluster, Singapore University of Technology and Design, 8 Somapah Road, Singapore 487372
}

(Received 24 January 2020; accepted 2 June 2020; published 22 June 2020)

\begin{abstract}
We propose protocols to prepare highly excited energy eigenstates of a trapped ion in a harmonic trap which do not use laser pulses to induce transitions among internal levels. Instead the protocols rely on smoothly deforming the trapping potential between single and double wells. The speed of the changes is set to minimize nonadiabatic transitions by keeping the adiabaticity parameter constant. High fidelities are found for times more than two orders of magnitude smaller than with linear ramps of the control parameter. Deformation protocols are also devised to prepare superpositions to optimize interferometric sensitivity, combining the ground state and a highly excited state.
\end{abstract}

DOI: 10.1103/PhysRevResearch.2.023372

\section{INTRODUCTION}

A trapped-ion architecture for quantum technologies rests on combining basic operations such as logic gates or shuttling, and generally needs fast and accurate control of internal and motional states. Preparing (Fock) states with a well defined number of vibrational quanta is one of the basic manipulations that may be used to implement quantum memories, entanglement operations, or communications $[1,2]$. Fock states with large number of phonons can be useful in metrology protocols based on NOON states, which give measurement outcomes with uncertainties reaching the Heisenberg bound [3,4]. Also, superpositions of eigenstates with maximally separated energies give optimal interferometric sensitivities [5,6], e.g., to measure motional frequency changes [7].

Several schemes to prepare Fock states have been proposed [3,8-14], but for a trapped ion they have only been produced stepwise by sequences of pulses, which is quite challenging for a large phonon number $n$. Resonant pulses need of the order of $n$ pulses with accurately defined frequency and area, so intensity and frequency errors and timing imperfections reduce the fidelity [15]. Adiabatic transfers admit parameter flexibility but the long times needed make them prone to suffer from decoherence and noise, which may be mitigated $[13,14]$ by shortcuts to adiabaticity (STA) $[16,17]$.

A recent experiment [7] applied sequences of Rabi pulses with unprecedented accuracy to approximately reach Fock states of up to $n=100$ transfering $50 \%$ of the population. To reach the highest Fock states, higher order sidebands, i.e., pulses that jump more than a single level at a time (up to four in this case), had to be applied, but still the required time and

Published by the American Physical Society under the terms of the Creative Commons Attribution 4.0 International license. Further distribution of this work must maintain attribution to the author(s) and the published article's title, journal citation, and DOI. errors grow rapidly with the phonon number, and the measurable gains in interferometric sensitivity using superpositions with the ground state were limited to $n \leqslant 12$.

While lasers will keep playing a major role in quantum technologies in the foreseeable future, a laser-based architecture does not scale well for complex operations involving large registers and/or many connected or repeated modulii. The large overhead of laser power and control $[18,19]$, instabilities in frequency, position or intensity, and spontaneous decay make engineering large scale devices challenging. This motivates the exploration of different, truly scalable platforms, e.g., using microwave control $[18,19]$ and microfabricated structures, which of course will involve their own, different, technical limitations. The goal pursued here is to create an excited Fock state for a single ion from the ground state without laser-induced internal transitions involved, by means of deformations of the trap. The potentials in linear, multielectrode Paul ion traps can be deformed by programming the voltages applied to the electrodes to control motional states, see Refs. [20-25]. A change of species implies a relatively minor software adaptation. This type of control can even be applied to other particles besides ions, like nanoparticles [26] or electrons [27].

The approach proposed is depicted in Fig. 1 and involves three steps: (a) demultiplexing; (b) bias inversion; (c) multiplexing. Steps (a) and (c) could be carried out adiabatically or using some shortcut to adiabaticity since the level ordering at the start and at the end of the process is conserved. For the second step the ordering of the levels is altered, so there is no global adiabatic mapping that connects initial and final states. However, in a fast process the wells are effectively independent and STA approaches can also be applied [28]. A faster-than-adiabatic approach for step (a) was worked out in Ref. [1] with neutral atoms, but only for a model with two motional levels. In this paper we design step (c) using an STA approach to minimize the nonadiabatic transitions distributing them homogeneously along the process time [29,30]. The first step requires a similar protocol but in reverse. 


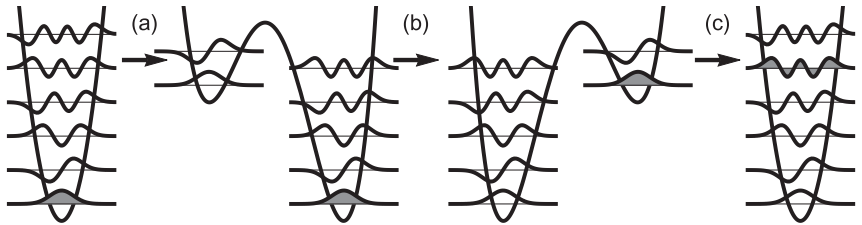

FIG. 1. Scheme for Fock-state preparation. (a) Demultiplexing: splitting the harmonic trap into an asymmetrical double well; (b) Bias inversion of the double well; (c) Multiplexing: inverse of demultiplexing. The shaded wave functions are the ideal initial and target states.

\section{MULTIPLEXING}

Consider a single ion in a trap that is effectively one dimensional driven by the Hamiltonian

$$
H(t)=\frac{p^{2}}{2 M}+\alpha(t) x^{2}+\beta(t) x^{4}+\gamma(t) x,
$$

where $x, p$ are the position and momentum operators, and $M$ is the mass of the ion; $\alpha(t), \beta(t)$, and $\gamma(t)$ are in principle time-dependent coefficients.

The trapping potential $V_{t}(x)=\alpha(t) x^{2}+\beta(t) x^{4}+\gamma(t) x$ is a double well potential when $\alpha(t)<0$ and $\beta(t)>0$. The term $\gamma(t) x$ corresponds to a homogeneous electric field that induces an energy bias between the wells. In the symmetric potential $(\gamma=0)$ the minima are at $x_{0, \pm}= \pm \sqrt{-\alpha /(2 \beta)}$. We consider the bias small enough so that the shift of the minima depends linearly on $\gamma$ [28],

$$
x_{0, \pm} \approx \pm \sqrt{-\alpha /(2 \beta)}+\gamma /(4 \alpha),
$$

valid when

$$
|\gamma| \ll 4 \sqrt{2} \sqrt{-\alpha^{3} / \beta} / 3,
$$

which defines the small-bias regime, in which the energy difference between the wells is approximately $\Delta V_{t}=\gamma D$, where

$$
D \equiv x_{0,+}-x_{0,-}=\sqrt{-2 \alpha / \beta}
$$

is the distance between the minima. The parameters for the initial double well will be chosen within this regime. The effective frequency $\omega_{\text {eff, }}$ of each well, in the small-bias regime is

$$
\omega_{\mathrm{eff}, \pm} \approx \Omega=2 \sqrt{-\alpha / M} .
$$

When $\alpha>0$ and $\beta=0$ the trapping potential is harmonic with angular frequency $\omega=\sqrt{2 \alpha / M}$. Multiplexing consists on driving the system from the double well configuration to the harmonic trap configuration so that the initial eigenstates are dynamically mapped onto the final ones. For simplicity we shall keep $\gamma(t)$ fixed, $\gamma(t)=\gamma$. The boundary conditions in a multiplexing operation are $\alpha_{0}<0, \beta_{0}>0$ for the initial values and $\alpha_{f}>0, \beta_{f}=0$ for the final values,

$$
\begin{aligned}
& V_{t=0}(x)=\alpha_{0} x^{2}+\beta_{0} x^{4}+\gamma x, \\
& V_{t=t_{f}}(x)=\alpha_{f}\left(x-x_{\mathrm{eq}}\right)^{2}-\gamma /\left(4 \alpha_{f}^{2}\right),
\end{aligned}
$$

with $x_{\text {eq }} \equiv-\gamma /\left(2 \alpha_{f}\right)$. We shall also impose that the frequency of the final harmonic trap is equal to the frequency of the initial wells so $\alpha_{f}=2\left|\alpha_{0}\right|$.
If the evolution is adiabatic, the lowest state of the upper well ( $n$th state globally) will become the $n$th Fock excited state $|n\rangle$ of the final harmonic potential. If the wells are deep enough, in the left (right) well there is a set of harmonic eigenstates $\left|n_{L}\right\rangle\left(\left|n_{R}\right\rangle\right)$ with energies $E_{n_{L}}=\hbar \Omega_{0}\left(n_{L}+\right.$ $1 / 2)\left(E_{n_{R}}=\hbar \Omega_{0}\left(n_{R}+1 / 2\right)+\Delta V_{t}\right)$, where $\Omega_{0} \equiv 2 \sqrt{-\alpha_{0} / m}$. We need the initial ground state of the right well, $\left|0_{R}\right\rangle$, to be the $n$th excited state of the whole system, so the inequality $E_{(n-1)_{L}}<E_{0_{R}}<E_{n_{L}}$ must be satisfied,

$$
n-1<\gamma D_{0} /\left(\hbar \Omega_{0}\right)<n,
$$

where $D_{0} \equiv D\left(\alpha_{0}, \beta_{0}\right)$. The ratio $D / \Omega=\sqrt{M /(2 \beta)}$ only depends on $\beta$ so a change of $\alpha$ within the small bias regime for constant $\beta$ does not modify this state ordering. In our simulations we choose the value

$$
\gamma=(n-1 / 2) \hbar \Omega_{0} / D_{0}
$$

for the bias. The small-bias condition and Eq. (7) provide an upper bound for the highest Fock state that can be prepared with $\alpha_{0}$ and $\beta_{0}$,

$$
n \ll 4 \sqrt{-M \alpha_{0}^{3} /\left(\hbar^{2} \beta_{0}^{2}\right)} / 3 .
$$

To design the driving of the control parameters, a straightforward approach would be an adiabatic evolution, for example a linear ramp protocol along a large run time. Long times, however, are inadequate for many applications and give rise to heating and decoherence. Shortcuts to adiabaticity $[16,17]$ stand out as a practical, faster option.

\section{DESIGN OF THE PROCESS}

Shortcuts to adiabaticity [16,17] are a family of methods that speed up adiabatic processes to get the same final populations or states in shorter times. Shortcuts have been applied for many different systems and operations and can be adapted to be robust against implementation errors and noise [17,31].

Among the different STA techniques available, Fast quasiadiabatic dynamics (FAQUAD) [29] is well suited to our current objective. Invariants-based inverse engineering [32] requires explicit knowledge of a dynamical invariant of the Hamiltonian, which is not available here, and fast-forward driving $[33,34]$ produces potentials with singularities due to the nodes of the wave function [35], which can be problematic with highly excited states. FAQUAD reduces the diabatic transitions between the states of the Hamiltonian by making the adiabaticity criterion constant during the process. For a time-dependent Hamiltonian that depends on a single control parameter $H(t)=H[\lambda(t)]$ such that $\lambda(t)$ is a monotonous function in the $\left[0, t_{f}\right]$ interval, the adiabaticity criterion to avoid transitions between the instantaneous eigenstates $|n(\lambda)\rangle$ and $|m(\lambda)\rangle$ is [36]

$$
\hbar \dot{\lambda}\left|\frac{\langle n(\lambda)|d H / d \lambda| m(\lambda)\rangle}{\left[E_{n}(\lambda)-E_{m}(\lambda)\right]^{2}}\right|=c \ll 1,
$$

where $E_{n}(\lambda)\left(E_{m}(\lambda)\right)$ are the instantaneous eigenenergies and the dot stands for time derivative.

FAQUAD imposes a constant $c$, so Eq. (10) becomes a differential equation for $\lambda(t)$. The value of $c$ is determined by the boundary conditions $\lambda(0)$ and $\lambda\left(t_{f}\right)$. Equation (10) 

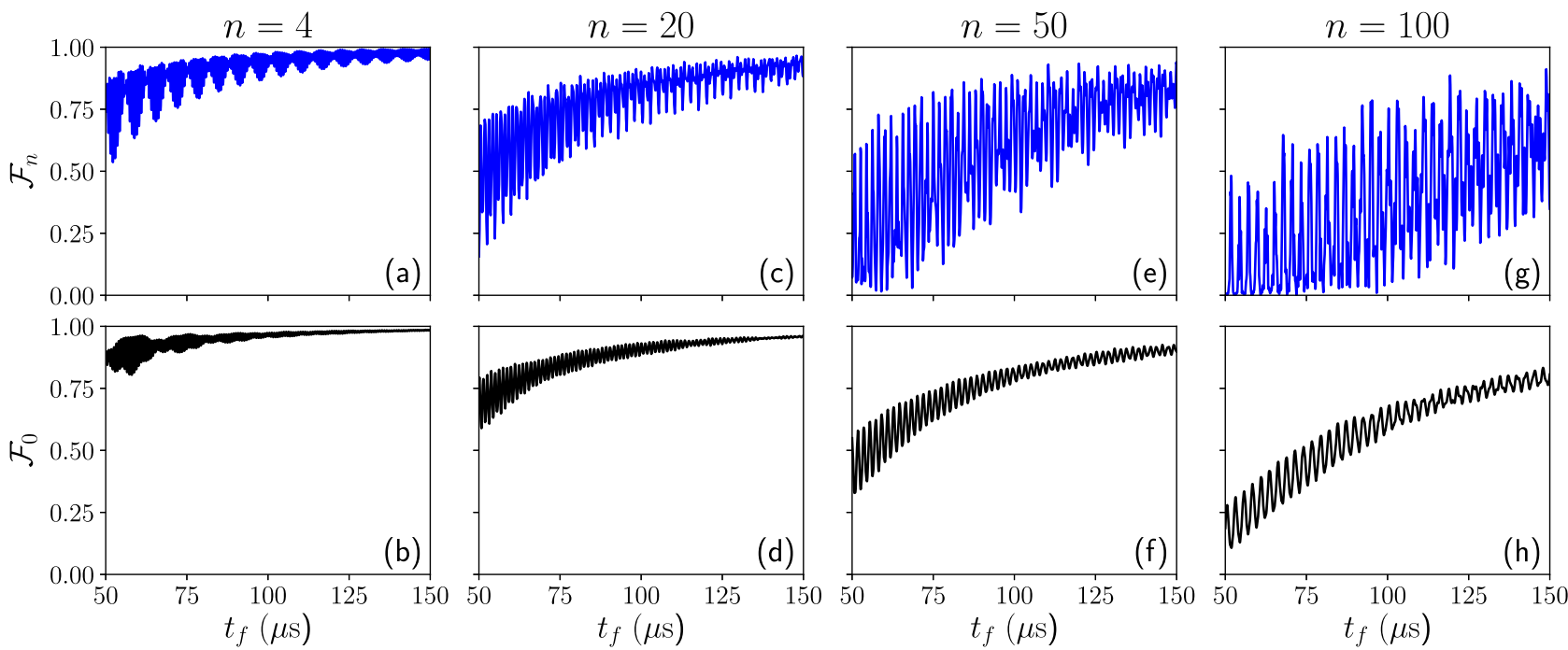

FIG. 2. Preparation of Fock states $|n\rangle$ and superposition states $\left(|0\rangle+e^{i \varphi}|n\rangle\right) / \sqrt{2}$ using FAQUAD. The different columns show the results for the different values of $n$. Upper row: fidelity of the stated evolved by FAQUAD from the $n$th excited of the double well with respect to $|n\rangle$, the $n$th Fock state of the harmonic trap. Lower row: fidelity of the state evolved from the ground state with respect to $|0\rangle$ using the FAQUAD trap deformation designed to get $|n\rangle . \alpha_{0}=-4.7 \mathrm{pN} / \mathrm{m}, \alpha_{f}=-2 \alpha_{0}=9.4 \mathrm{pN} / \mathrm{m}, \beta_{0}=0.052 \mathrm{~N} / \mathrm{m}^{3}, \beta_{f}=0, M\left({ }^{9} \mathrm{Be}^{+}\right)=9.012$ a.u., $\epsilon=1 \mathrm{pN} / \mathrm{m}$, and $\kappa=100 /\left(\alpha_{0}-\alpha_{f}\right)=-7.092 \mathrm{~m} / \mathrm{pN}$.

implies that the control parameter evolves more slowly when the Hamiltonian changes rapidly with the control parameter and/or near avoided crossings.

We eliminate one degree of freedom in Eq. (1) by taking $\alpha$ as the master control parameter $(\alpha=\lambda)$ and making $\beta=$ $\beta(\alpha)$. Equation (10) has to be solved with the boundary conditions for $\alpha$ and $\beta$. To choose $\beta(\alpha)$ we consider that the largest possible values of $\beta$ should hold while $\alpha$ changes sign so that the levels in the intermediate quartic well are not too close. A simple choice is to keep $\beta \approx \beta_{0}$ constant until $\alpha>0$ increases and the quadratic part dominates. Then we can let $\beta$ drop to zero without any significant effect. While $\beta$ is constant the energy difference between the wells in units of the instantaneous motional quantum

$$
N_{q}=\gamma D /(\hbar \Omega)=\gamma \sqrt{M /(2 \beta)} / \hbar,
$$

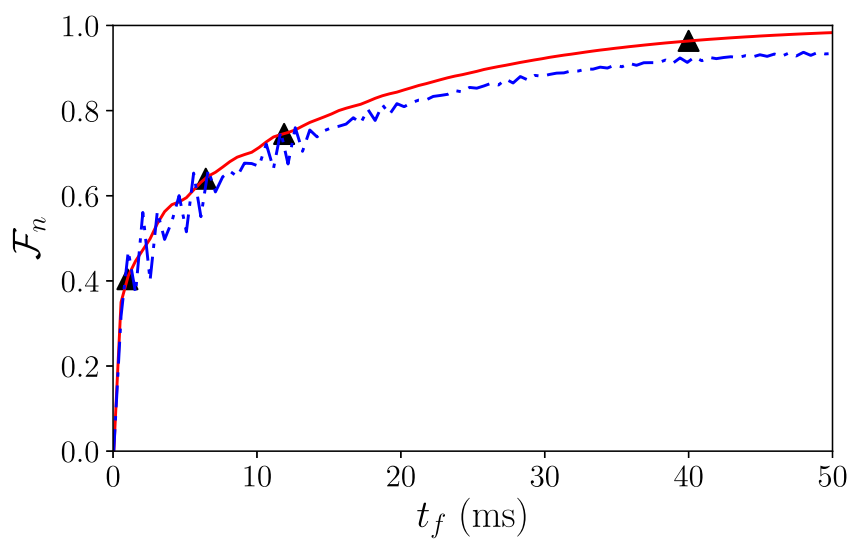

FIG. 3. Fidelity vs final time for the creation of Fock states using a linear ramp of the control parameter $\alpha$ : triangles, $n=20$; solid red line, $n=50$ and dashed blue line $n=100$. Same parameters as in Fig. 2. is constant. We choose for $\beta$ the form

$$
\beta(\alpha)=a+b S[\kappa(\alpha-\epsilon)],
$$

where $S(x)=\left(1+e^{-x}\right)^{-1}$ is the (sigmoid) logistic function. $\epsilon$ and $\kappa$ set the position and the width of the region where the parameter $\beta$ ramps from its initial to the final value. We choose $\epsilon>0$ so that $\beta \approx \beta_{0}$ around $\alpha=0$. A larger $\kappa$ implies a narrower jump. When $\kappa \gg \max \left\{\left(\left|\alpha_{0}\right|+\epsilon\right)^{-1},\left(\left|\alpha_{f}\right|-\epsilon\right)^{-1}\right\}$ the ramp of $\beta$ is narrow enough so that when $\alpha$ goes to $\alpha_{0}$ $\left(\alpha_{f}\right) \beta$ goes asymptotically to $a(a+b)$ and then the boundary conditions demand that $a=-b=\beta_{0}$.

We choose $\alpha_{0}=-4.7 \mathrm{pN} / \mathrm{m}, \alpha_{f}=2\left|\alpha_{0}\right|=9.4 \mathrm{pN} / \mathrm{m}$, $\beta_{0}=0.052 \mathrm{~N} / \mathrm{m}^{3}$, and ${ }^{9} \mathrm{Be}^{+}$ions in the numerical simulations, so $\Omega_{0}=2 \pi \times 5.6 \mathrm{MHz}$ and $D_{0}=13.45 \mu \mathrm{m}$. For the ground state of the highest energy well to be the $n$th excited state of the full Hamiltonian, the bias is chosen as $\gamma=(n-$ $0.5) \hbar \Omega_{0} / D_{0}$. Wilson et al. [37] implemented $\alpha_{0}$ values in this range and $\beta_{0}$ values five times smaller. The necessary increase may be achieved, keeping the same trap geometry, by a $3 / 4$ reduction of effective trap size accompanied by electrode cooling or surface treatment to avoid heating $[20,38,39]$, and a feasible slight increase by 1.2 of applied voltages [40], or, alternatively, by a change in the geometry with the same sizes and voltages [41]. These $\alpha_{0}$ and $\beta_{0}$ imply a resolution for $\gamma$ in the sub zN range to select a specific $n$, for which calibrations have been described $[42,43]$. The stability with respect to small errors in $\gamma$ is analyzed in the Appendix. Spurious, slow drifts in this constant force were identified as a main technical issue to implement double barriers [40,44], but they may be compensated [40].

Avoided level crossings occur at $\alpha<0$, near $\alpha=0$ in a critical region where the small bias condition fails and the double well becomes a single quartic well. The gap between the eigenstates near $\alpha=0$ is approximately proportional to $\beta^{1 / 3}$ [45]. Thus, at $\alpha \approx 0, \beta$ should be as large as possible within experimental constraints. 


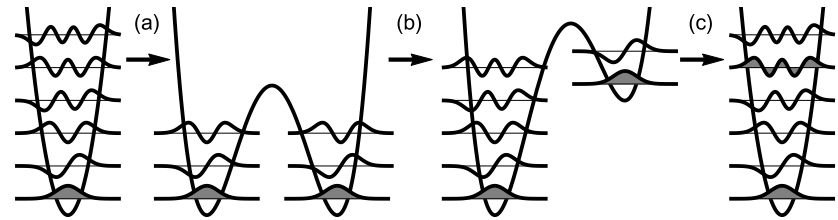

FIG. 4. Scheme to prepare superpositions of the ground state and a Fock state of the harmonic oscillator, $\left(|0\rangle+e^{i \varphi}|n\rangle\right) / \sqrt{2}$. (a) Splitting of the ground state; (b) Biasing; (c) Merging the two wells into a harmonic trap. The shaded wave functions are the ideal initial and target states.

In our multilevel scenario we modify Eq. (10) to [30]

$$
c=\hbar \dot{\lambda} \sum_{m \neq n}\left|\frac{\langle n(\lambda)|d H / d \lambda| m(\lambda)\rangle}{\left[E_{n}(\lambda)-E_{m}(\lambda)\right]^{2}}\right|,
$$

taking only the four closest eigenstates (two from below and two from above) of the relevant state in the sum. Note the shorthand notation $|n\rangle \equiv\left|n\left(\lambda_{f}\right)\right\rangle$ for the eigenstates of the final harmonic oscillator.

\section{RESULTS}

We have numerically solved the time-dependent Schrödinger equation driven by Eq. (1). We compare the performance of the protocols designed using FAQUAD with a linear ramp of the control parameter $\alpha(t)$, using the same $\beta(\alpha)$ as for FAQUAD.

The upper row of Fig. 2 shows the results of the multiplexing step [(c) in Fig. 1] for different $n$. The fidelity is $\mathcal{F}_{n}=\left|\left\langle n \mid \psi_{n}^{F}\right\rangle\right|$, where $\left|\psi_{n}^{F}\right\rangle$ is the final state after FAQUAD evolution. The fidelity if $\alpha(t)$ follows a linear ramp is depicted in Fig. 3. FAQUAD attains fidelities above $\mathcal{F}=0.9$ for final times of less than $100 \mu \mathrm{s}$, while the linear ramp needs evolution times up to $50 \mathrm{~ms}$ for similar fidelities. In Fig. 2 (upper panels) the maximum fidelities for similar final times decrease and the width of the fidelity oscillations increases for larger $n$. Both effects can be mitigated using a local adiabatic approach, see the final discussion. Nevertheless, for the studied final times, fidelities above $\mathcal{F}=0.9$ for $n=100$ can be reached for specific values of $t_{f}$. In Ref. [7] a table shows the final times required to create each Fock state by combining Rabi pulses. For $n=4$ only $38 \mu$ s are needed, but for $n=100$ the total time grows to $335 \mu$ s, even though higher order sidebands were applied. In comparison, even if the times needed are orders of magnitude larger, the
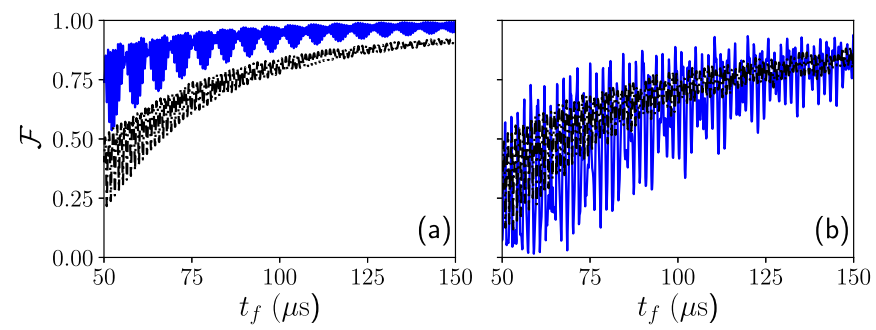

FIG. 5. Fidelities after performing the multiplexing process using FAQUAD (solid blue lines) and LA method: (a) $n=4$ and (b) $n=50$. Same parameters as in Fig. 2. remarkable stability of the fidelity curve with respect to $n$ is noteworthy for the linear ramp in Fig. 3. This stability of a trap deformation method also holds, although somewhat weakened, in the upper edge of the fidelity curve using FAQUAD, which is experimentally accessible since time resolutions of $20 \mathrm{~ns}$ have been demonstrated [25].

The explanation for the decreasing fidelities as the process aims at higher Fock states is that the nearest energy levels get closer, and transitions among more and more levels occur making the interference pattern, inherent in FAQUAD [28], more complex.

\section{PREPARING SUPERPOSITIONS}

The protocols studied so far also allow us to prepare states $\left|\psi_{\varphi}\right\rangle=\left(|0\rangle+e^{i \varphi}|n\rangle\right) / \sqrt{2}$ up to a relative phase $\varphi$. A modification of the sequence in Fig. 1 leads to superposition states, see Fig. 4. The success of the protocol [step (c)] is measured with the fidelity $\mathcal{F}_{n}$ to reach $|n\rangle$ starting in the $n$th excited state of the double well, and the fidelity $\mathcal{F}_{0}(n)$ to reach $|0\rangle$ starting in the ground state while using the deformation devised to reach $|n\rangle$. (The average $\left(\mathcal{F}_{0}(n)+\mathcal{F}_{n}\right) / 2$ is the maximal fidelity with respect to the states labeled by $\varphi$ ). The upper row of Fig. 2 pictures $\mathcal{F}_{n}$ for $n=4,20,50,100$ and the lower panels the corresponding $\mathcal{F}_{0}(n)$. The $\mathcal{F}_{0}(n)$ are remarkably close to the $\mathcal{F}_{n}$, which makes superpositions $\left|\psi_{\varphi}\right\rangle$ feasible with high fidelity.

In Ref. [7] these superpositions were created via Rabi pulses for measuring deviations from a nominal trap frequency. The maximum sensitivity was reached for the superposition of the ground and the $n=12$ states.

\section{DISCUSSION}

We have proposed to prepare highly excited Fock states and superpositions with the ground state in trapped ions using deformations between double and single wells. Since no Rabi pulses are involved, these protocols can be applied to different atomic species or particles.

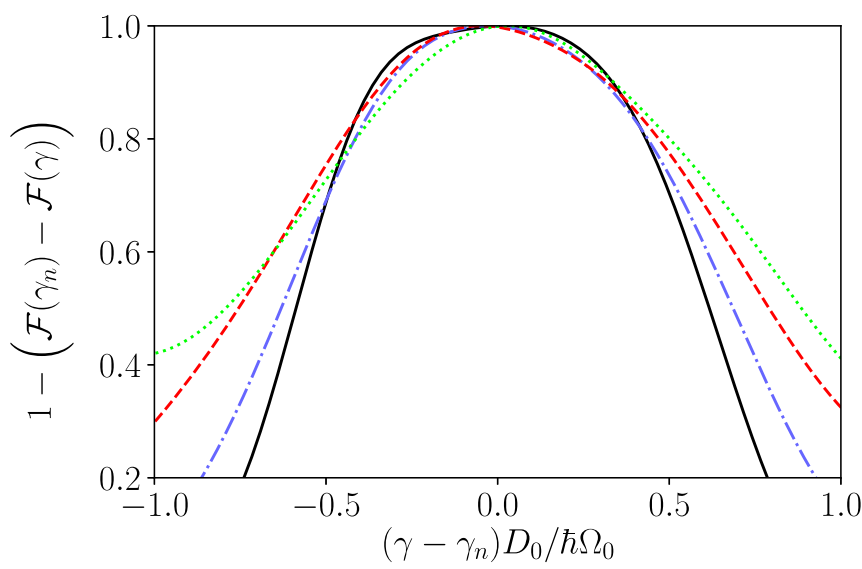

FIG. 6. Change of the fidelity against variations of the bias term with respect to the ideal value for the Fock-state preparation protocols in the main text: $n=4$, solid black line; $n=20$, dot-dashed blue line; $n=50$, dashed red line; $n=100$, dotted green line. Same parameters as in Fig. 2 of the main text. 
TABLE I. Evolution times at fidelity maxima and fidelity errors around them for different errors in $\gamma$. Same parameters as in Fig. 2 of the main text.

\begin{tabular}{|c|c|c|c|c|c|c|}
\hline \multirow[b]{2}{*}{$n$} & \multirow[b]{2}{*}{$t_{f}(\mu \mathrm{s})$} & \multirow[b]{2}{*}{$\mathcal{F}\left(\gamma_{n}\right)$} & \multirow[b]{2}{*}{$\sigma_{n}\left(\mathrm{zN}^{-2}\right)$} & \multicolumn{3}{|c|}{$\mathcal{F}(\gamma)-\mathcal{F}\left(\gamma_{n}\right)$} \\
\hline & & & & $\delta \gamma=0.001 \mathrm{zN}$ & $\delta \gamma=0.01 \mathrm{zN}$ & $\delta \gamma=0.1 \mathrm{zN}$ \\
\hline 4 & 149.60 & 0.9928 & -16.2 & $-8.1 \times 10^{-6}$ & $-8.1 \times 10^{-4}$ & $-8.1 \times 10^{-2}$ \\
\hline 20 & 147.90 & 0.9697 & -20.9 & $-1.0 \times 10^{-5}$ & $-1.0 \times 10^{-3}$ & $-1.0 \times 10^{-1}$ \\
\hline 50 & 149.89 & 0.9334 & -19.1 & $-9.6 \times 10^{-6}$ & $-9.6 \times 10^{-4}$ & $-9.6 \times 10^{-2}$ \\
\hline 100 & 148.90 & 0.9150 & -30.7 & $-1.5 \times 10^{-5}$ & $-1.5 \times 10^{-3}$ & $-1.5 \times 10^{-1}$ \\
\hline
\end{tabular}

A FAQUAD approach which distributes diabatic transitions homogeneously through all the process provides a significant speedup with respect to a linear ramp of the control parameter. Methods similar in spirit to FAQUAD may also be applied [17]. The local adiabatic (LA) method [46] only uses the instantaneous energy gap between the eigenstates to modulate the rate of change of the control parameter. Adapted to our multilevel scenario, we set

$$
c_{\mathrm{LA}}=\hbar \dot{\lambda}_{\mathrm{LA}} \sum_{m \neq n}\left[E_{n}(\lambda)-E_{m}(\lambda)\right]^{-2},
$$

as a constant given by the boundary conditions, and parametrize $\beta(\alpha)$ as before. We compare the performance of FAQUAD and LA in Fig. 5. For small $n$ FAQUAD outperforms LA, but LA is more stable as $n$ increases, due to a lesser role of quantum interferences [29].

This paper demonstrates the potential of trap deformations to control motional states. Future work could be to find protocols for ion chains, to make full use of the dimensionality of the parameter space [47], reduced here to one for simplicity, or to look for smooth, doable functional forms for the time dependence of the trap increasing the minimal gap, combined with numerical optimization of the deformation.

\section{ACKNOWLEDGMENTS}

We acknowledge discussions with J. Alonso. This work was supported by the Basque Country Government (Grant No. IT986-16) and PGC2018-101355-B-I00 (MCIU/AEI/FEDER,UE). M.P. acknowledges support from the Singapore Ministry of Education, Singapore Academic Research Fund Tier-II (MOE2018-T2-2-142). M.A.S. acknowledges support by the Basque Government predoctoral program (Grant No. PRE-2019-2-0234).

\section{APPENDIX: STABILITY OF THE PROTOCOL WITH RESPECT TO ERRORS IN THE BIAS TERM}

The fixed (bias) force $\gamma$ has to be set with enough precision to discriminate among different $n$ values.
As explained in the main text, to produce the Fock state number $n$ we choose $\gamma=\gamma_{n} \equiv(n-1 / 2) \hbar \Omega_{0} / D_{0}$. If the bias force has an error $\delta \gamma$ with respect to $\gamma_{n}$, the error in the Fock state number (treated as a continuous variable) is

$$
\Delta_{n}=D_{0} \delta \gamma / \hbar \Omega_{0}=\frac{1}{\hbar} \sqrt{M / 2 \beta} \delta \gamma
$$

We need $\Delta_{n}<1$, which gives an upper bound for $\delta \gamma<\Delta \gamma \equiv$ $\hbar \sqrt{\frac{2 \beta}{M}}$. For $\beta=0.052 \mathrm{~N} / \mathrm{m}^{3}$ and beryllium ions, $\Delta \gamma=0.278$ $\mathrm{zN}$. Forces can be measured and calibrated with this precision [42,43,48-50].

To analyze how an error in the bias with respect to $\gamma_{n}$ could deteriorate the fidelity of the STA-accelerated multiplexing process, we have made simulations for the values of $n$ in the main text, $n=4,20,50,100$. The final time $t_{f}$ for each $n$ is chosen at the maximum fidelity below $150 \mu \mathrm{s}$ in Fig. 2 of the main text. Detailed information of the final times and fidelities can be found in Table I. Figure 6 shows the fidelities, normalized to one when $\gamma=\gamma_{n}$. Two interesting features are (i) all curves are quite flat in the interval $\left|\gamma-\gamma_{n}\right| \leqslant \hbar \Omega_{0} / 2 D_{0}$, and (ii) neither the flatness nor the actual value of the maximum fidelity, see the third column in Table I, deteriorate too rapidly when increasing $n$.

The fidelity depends on a small error $\delta \gamma$ as

$$
\begin{aligned}
\mathcal{F}(\gamma) & =\mathcal{F}\left(\gamma_{n}+\delta \gamma\right) \\
& =\mathcal{F}\left(\gamma_{n}\right)+\left.\delta \gamma \frac{\partial \mathcal{F}}{\partial \gamma}\right|_{\gamma=\gamma_{n}}+\left.\frac{1}{2} \delta \gamma^{2} \frac{\partial^{2} \mathcal{F}}{\partial \gamma^{2}}\right|_{\gamma=\gamma_{n}} .
\end{aligned}
$$

We may disregard the linear term at a peak of the fidelity (i.e., for a process time for which $\left.\frac{\partial \mathcal{F}}{\partial \gamma}\right|_{\gamma=\gamma_{n}}=0$ ) or also when averaging over unbiased deviations, i.e., assuming that $\delta \gamma$ may be treated as an unbiased random number with zero mean. In both cases the quadratic coefficient $\sigma_{n}=\left.\frac{\partial^{2} \mathcal{F}}{\partial \gamma^{2}}\right|_{\gamma=\gamma_{n}}$ measures the flatness of the fidelity and gives a measure of robustness. Table I gives $\sigma_{n}$, as well as $\mathcal{F}(\gamma)-\mathcal{F}\left(\gamma_{n}\right)$ for bias errors spanning different orders of magnitude around a fidelity peak.
[1] S. Martínez-Garaot, E. Torrontegui, X. Chen, M. Modugno, D. Guéry-Odelin, S.-Y. Tseng, and J. G. Muga, Phys. Rev. Lett. 111, 213001 (2013).

[2] C. Galland, N. Sangouard, N. Piro, N. Gisin, and T. J. Kippenberg, Phys. Rev. Lett. 112, 143602 (2014).
[3] J. Zhang, M. Um, D. Lv, J.-N. Zhang, L.-M. Duan, and K. Kim, Phys. Rev. Lett. 121, 160502 (2018).

[4] V. Giovannetti, S. Lloyd, and L. Maccone, Nat. Photonics 5, 222 (2011).

[5] N. Margolus and L. B. Levitin, Physica D 120, 188 (1998). 
[6] C. M. Caves and A. Shaji, Opt. Commun. 283, 695 (2010).

[7] K. C. McCormick, J. Keller, S. C. Burd, D. J. Wineland, A. C. Wilson, and D. Leibfried, Nature (London) 572, 86 (2019).

[8] J. I. Cirac, R. Blatt, A. S. Parkins, and P. Zoller, Phys. Rev. Lett. 70, 762 (1993).

[9] J. I. Cirac, R. Blatt, and P. Zoller, Phys. Rev. A 49, R3174(R) (1994).

[10] D. M. Meekhof, C. Monroe, B. E. King, W. M. Itano, and D. J. Wineland, Phys. Rev. Lett. 76, 1796 (1996).

[11] L. Davidovich, M. Orszag, and N. Zagury, Phys. Rev. A 54, 5118 (1996).

[12] R. L. de Matos Filho and W. Vogel, Phys. Rev. Lett. 76, 4520 (1996).

[13] O. Abah, R. Puebla, and M. Paternostro, Phys. Rev. Lett. 124, 180401 (2020).

[14] M. Um, J. Zhang, D. Lv, Y. Lu, S. An, J.-N. Zhang, H. Nha, M. S. Kim, and K. Kim, Nat. Commun. 7, 11410 (2016).

[15] I. E. Linington, P. A. Ivanov, N. V. Vitanov, and M. B. Plenio, Phys. Rev. A 77, 063837 (2008).

[16] E. Torrontegui, S. Ibáñez, S. Martínez-Garaot, M. Modugno, A. del Campo, D. Guéry-Odelin, A. Ruschhaupt, X. Chen, and J. G. Muga, in Advances in Atomic, Molecular, and Optical Physics, Advances in Atomic, Molecular, and Optical Physics, Vol. 62, edited by P. R. B. Ennio Arimondo and C. C. Lin (Academic, New York, 2013), pp. 117-169.

[17] D. Guéry-Odelin, A. Ruschhaupt, A. Kiely, E. Torrontegui, S. Martínez-Garaot, and J. G. Muga, Rev. Mod. Phys. 91, 045001 (2019).

[18] C. Ospelkaus, C. E. Langer, J. M. Amini, K. R. Brown, D. Leibfried, and D. J. Wineland, Phys. Rev. Lett. 101, 090502 (2008).

[19] B. Lekitsch, S. Weidt, A. G. Fowler, K. Mølmer, S. J. Devitt, C. Wunderlich, and W. K. Hensinger, Sci. Adv. 3, e1601540 (2017).

[20] H. Kaufmann, T. Ruster, C. T. Schmiegelow, F. Schmidt-Kaler, and U. G. Poschinger, New J. Phys. 16, 073012 (2014).

[21] J. P. Home and A. M. Steane, Quantum Info. Comput. 6, 289 (2006).

[22] A. H. Nizamani and W. K. Hensinger, Appl. Phys. B 106, 327 (2012).

[23] H. A. Fürst, M. H. Goerz, U. G. Poschinger, M. Murphy, S. Montangero, T. Calarco, F. Schmidt-Kaler, K. Singer, and C. P. Koch, New J. Phys. 16, 075007 (2014).

[24] A. Walther, F. Ziesel, T. Ruster, S. T. Dawkins, K. Ott, M. Hettrich, K. Singer, F. Schmidt-Kaler, and U. Poschinger, Phys. Rev. Lett. 109, 080501 (2012).

[25] R. Bowler, J. Gaebler, Y. Lin, T. R. Tan, D. Hanneke, J. D. Jost, J. P. Home, D. Leibfried, and D. J. Wineland, Phys. Rev. Lett. 109, 080502 (2012).
[26] W. Guan, S. Joseph, J. H. Park, P. S. Krstić, and M. A. Reed, Proc. Natl. Acad. Sci. 108, 9326 (2011).

[27] D. Segal and M. Shapiro, Nano Lett. 6, 1622 (2006).

[28] S. Martínez-Garaot, M. Palmero, D. Guéry-Odelin, and J. G. Muga, Phys. Rev. A 92, 053406 (2015).

[29] S. Martínez-Garaot, A. Ruschhaupt, J. Gillet, T. Busch, and J. G. Muga, Phys. Rev. A 92, 043406 (2015).

[30] M. Palmero, M. A. Simón, and D. Poletti, Entropy 21, 1207 (2019).

[31] A. Ruschhaupt, X. Chen, D. Alonso, and J. G. Muga, New J. Phys. 14, 093040 (2012).

[32] X. Chen, E. Torrontegui, and J. G. Muga, Phys. Rev. A 83, 062116 (2011).

[33] S. Masuda and K. Nakamura, Proc. R. Soc. London A 466, 1135 (2010).

[34] E. Torrontegui, S. Martínez-Garaot, A. Ruschhaupt, and J. G. Muga, Phys. Rev. A 86, 013601 (2012).

[35] S. Martínez-Garaot, M. Palmero, J. G. Muga, and D. GuéryOdelin, Phys. Rev. A 94, 063418 (2016).

[36] L. I. Schiff, Quantum Mechanics (McGraw-Hill, New York, 1968).

[37] A. C. Wilson, Y. Colombe, K. R. Brown, E. Knill, D. Leibfried, and D. J. Wineland, Nature (London) 512, 57 (2014).

[38] M. Brownnutt, M. Kumph, P. Rabl, and R. Blatt, Rev. Mod. Phys. 87, 1419 (2015)

[39] C. D. Bruzewicz, J. Chiaverini, R. McConnell, and J. M. Sage, Appl. Phys. Rev. 6, 021314 (2019).

[40] T. Ruster, C. Warschburger, H. Kaufmann, C. T. Schmiegelow, A. Walther, M. Hettrich, A. Pfister, V. Kaushal, F. SchmidtKaler, and U. G. Poschinger, Phys. Rev. A 90, 033410 (2014).

[41] F. N. Krauth, J. Alonso, and J. P. Home, J. Phys. B 48, 015001 (2015).

[42] M. J. Biercuk, H. Uys, J. W. Britton, A. P. VanDevender, and J. J. Bollinger, Nat. Nanotech. 5, 646 (2010).

[43] P. A. Ivanov, Phys. Scr. 95, 025103 (2020).

[44] M. Palmero, S. Martínez-Garaot, U. G. Poschinger, A. Ruschhaupt, and J. G. Muga, New J. Phys. 17, 093031 (2015).

[45] M. Vranivcar and M. Robnik, Prog. Theoret. Phys. Suppl. 139, 214 (2000).

[46] J. Roland and N. J. Cerf, Phys. Rev. A 65, 042308 (2002).

[47] A. T. Rezakhani, W.-J. Kuo, A. Hamma, D. A. Lidar, and P. Zanardi, Phys. Rev. Lett. 103, 080502 (2009).

[48] R. Maiwald, D. Leibfried, J. Britton, J. C. Bergquist, G. Leuchs, and D. J. Wineland, Nat. Phys. 5, 551 (2009).

[49] T. F. Gloger, P. Kaufmann, D. Kaufmann, M. T. Baig, T. Collath, M. Johanning, and C. Wunderlich, Phys. Rev. A 92, 043421 (2015).

[50] R. Shaniv and R. Ozeri, Nat. Commun. 8, 14157 (2017). 